\title{
Challenges of moving theranostic nanomedicine into the clinic
}

\author{
Deepika Singh ${ }^{1}$, Fahima Dilnawaz ${ }^{1} \&$ Sanjeeb Kumar Sahoo*,1 \\ ${ }^{1}$ Nanomedicine Laboratory, Institute of Life Sciences, Nalco Square, Bhubaneswar, Odisha, 751023, India \\ *Author for correspondence: Tel.: +91 0674230 4341; sanjeebsahoo2005@gmail.com
}

\section{"Nanotheranostics is an add-on concept in nanomedicine and could find numerous application-oriented opportunities in clinics for disease management."}

First draft submitted: 24 October 2019; Accepted for publication: 18 November 2019; Published online: 6 January 2020

Keywords: diagnosis $\bullet$ drug delivery $\bullet$ imaging $\bullet$ nanomedicine $\bullet$ regulatory governance $\bullet$ theranostic

Increasing demand for a new strategy for disease treatment requires cautious coordination of diagnosis and therapy. Advances in nanotechnology have led to the integration of therapeutics and diagnostics in a single nanoscale agent referred to as theranostics [1]. This field has the potential to have major applications in clinics, such as improving specificity of targeted delivery, achieving efficient gene transfection and enhancing medical imaging, all to ameliorate treatment outcomes. In the case of cancer, the current treatment modalities cannot address the issue of involvement of multiple barriers (such as drug resistance, tumor microenvironment) in its growth and progression so delivering an effective treatment to the tumor site is a complicated and demanding task.

Nanomedicines are in the preliminary stage of clinical development, for example, the pharmaceutical company Cristal Therapeutics is currently undertaking a Phase I clinical trial for CriPec ${ }^{\circledR}$ docetaxel combined with the imaging agent Zirconium-89 for PET imaging. This is to evaluate the biodistribution and tumor accumulation of the nanomedicine in solid tumors with the aim of developing a better targeted therapy. Another company, Nanobiotix is conducting Phase I/II trials for NBTXR3 ${ }^{\circledR}$ comprising of hafnium oxide nanoparticles as a radio-enhancer to destroy tumor utilizing radiation-stimulated technology [2]. The impact of nanomedicines on health spending and cost-effectiveness is still difficult to predict because of the involvement of public and private funding [3]. Clinical treatment needs improvement in order to reduce the cost and side effects of the treatment. Moreover, alternative treatment options to painful surgeries are urgently needed. Presently, these advanced theranostic nanomedicines are confined to academic settings and are surrounded by many clinical challenges that need to be overcome before they can undergo bench to bedside transition.

\section{Key concepts of theranostic nanomedicines}

The most common and crucial theme in clinics, especially for cancer management is to detect the disease at its earliest possible premalignant state and to provide specific therapy against it. In achieving this, theranostic nanomedicine holds great promise. The advantageous aspects of nanoformulations are their ability to be absorbed and their high bioavailability, which enables reduction of doses to achieve the desired effect at the target site. Theranostic nanomedicines come in many different formats and structures. They can be composed of inorganic nanoparticles made of gold, silver, silica or magnetic nanoparticles, which act as imaging agents. They can then have different drugs either entrapped within them, or the nanoparticle shell can be conjugated to therapeutic agents such as drugs, ligands or antibodies. This allows simultaneous imaging and therapy [4]. Besides this, popular drug-delivery systems like liposomes, micelles, polymeric nanoparticles, solid lipid nanoparticles and dendrimers can be co-loaded with therapeutic and contrast agents in a single platform to form theranostic nanomedicine [5].

The contrast agent used must be compatible with already established diagnostic tools such as x-ray, computed tomography (CT), MRI and positron emission tomography (PET) or single-photon emission CT [6]. Various inorganic nanoparticles are in use as contrast agents because they possess free electrons and its excitation phase is utilized in various imaging modalities. For example, iron oxide nanoparticles are clinically accepted and used in

Future Medicine 
MRI because of their superparamagnetic nature. Indeed, an early Phase I clinical trial (NCT01895829) analyzing the efficacy of ferumoxytol as a contrast agent for imaging head and neck cancer is currently underway. Colloidal semiconductor nanoparticles such as quantum dots are widely used for live-cell imaging and in vivo diagnostics. For example, poly(ethylene glycol) based $\mathrm{CuInS}_{2} @ \mathrm{ZnS:Mn} \mathrm{quantum} \mathrm{dots} \mathrm{demonstrated} \mathrm{potential} \mathrm{as} \mathrm{dual} \mathrm{modality}$ probes for fluorescence/MRI of tumors in vivo [7]. Luminescent porous silicon nanoparticles are also used as a contrast agent for in vivo imaging [8]. With both therapeutic and imaging functionalities, theranostic nanomedicines hold great promise for simultaneous noninvasive diagnosis and treatment of disease. They also provide exciting possibilities for day-to-day real-time monitoring of drug release, distribution and treatment response of therapy in patients, not only for cancer but also for several other diseases [9]. Theranostic nanomedicines show supremacy ranging from patient stratification, monitoring intratumoral drug distribution, imaging-guided focal therapy and therapy response monitoring [1]. Nanotheranostics can also prolong systemic circulation, escape the host's immune system, reduce off-target effects and provide sustained release of drug and diagnostic agents at the disease site. Recently developed nanotheranostics also present the possibility of multifunctional bioimaging where nanoparticles can be simultaneously used for CT and MRI along with therapies such as photothermal therapy and radiotherapy for tumor treatment both in vitro and in vivo [10]. Currently, nanotheranostics have generated innovative strategies and have reached clinics to mitigate cardiovascular diseases and conditions such as thrombus. Thrombus is mainly composed of activated platelets and fibrin that obstructs arteries and veins leading to various life-threatening diseases. Recently, Jung et al. developed molecularly engineered theranostic nanoparticles, which can provide $\mathrm{H}_{2} \mathrm{O}_{2}$ triggered photoacoustic, signal amplification showing antithrombotic properties, which could be beneficial for the treatment of thrombosed vessels [11].

\section{Challenges for translation of theranostic nanoparticles into clinics Biological challenges}

Theranostic nanomedicine shows vast potential to improve human health by giving insights into the prevention, diagnosis and treatment of diseases. Despite considerable technological advancement in this direction, nanotheranostics becoming a new paradigm for disease diagnosis and treatment in clinics is still in its infancy. One of the major challenges associated with the translation of theranostic nanomedicine to clinics is the nano-bio interaction. The potential toxicity or incompatibility of the nanomedicine upon interaction with biological material can generate disorders like immunoreaction, inflammation or related diseases. The toxic effect is greatly dependent upon various parameters such as size, $\zeta$-potential and solubility of the nanoformulations [12]. When nanoparticles enter a biological system they interact with proteins, which leads to the formation of 'corona' on its surface. This adsorption of protein onto the surface of nanoparticles alters their size, stability, dispersibility, pharmacokinetics, biodistribution and toxicity profile $[13,14]$. In addition, published reports show that complement activation-related pseudo allergy is an acute adverse immune reaction caused by many nanoplatforms [15,16]. Therefore, the study of physicochemical characteristics of nanomedicines with respect to pathophysiology and heterogeneity of human diseases is quite essential. Moreover, the concept of a one-size-fits-all approach in the case of theranostic nanomedicine can make it difficult to get clinical approval, as the treatment varies from person to person [17]. Experimental evidence suggests that nanoparticles which have good therapeutic properties may not necessarily be good diagnostic tools. A tumor uptake and tumor visualization study performed with anti-EGFR coated gold nanoparticles with $20 \mathrm{~nm}$ size showed high tumor uptake whereas, gold nanoparticles with $50 \mathrm{~nm}$ size illustrated highest CT contrast enhancement. The above study suggested that size-dependent distribution of theranostic nanomedicines in tumor limits its use as a theranostic agent [18]. Therefore, the safety profile of nanotheranostics in humans remains a major concern for which long term close monitoring of patients in both early and advanced phases of clinical trials is needed.

\section{Commercialization challenges}

Another major challenge associated with the clinical translation of theranostic nanomedicines is the difficulty in formulating a controllable and reproducible synthesis process. Large-scale synthesis of nanoparticles suffers from insufficient batch-to-batch reproducibility, varied physical and chemical characteristics and low yield. Pharmaceutical manufacturing centers around quality and cost and it has been noticed that nanoplatforms with laborious and complex manufacturing processes rarely find their way into the clinic due to inconvenience caused to pharmaceutical companies $[17,19]$. Since theranostic nanoparticles comprise of a multifunctional unit, more precise chemistry, manufacturing and control along with good manufacturing practice are needed while translating from laboratory to clinics, this is difficult on a large scale. 
A third major issue that needs to be addressed is the wide gap between the scientific community and regulatory authorities. The commercialization of nanomedicine is being monitored by many government policies based on regulatory factors related to quality control, manufacturing practices, safety profile and patent protection. Timely and effective translation of theranostics to market is highly affected due to the deficiency of clear regulatory and safety guidelines [20,21]. Nanomedicines currently available on the market have passed the general regulatory standards, but these standards may not be sufficient and need further revision to confirm the quality, safety and efficacy of other nanotheranostics for human use.

\section{Strategies to overcome these challenges}

To overcome the biological barriers of nanotheranostics, much research effort needs to be dedicated to understanding the correlation of patient biology and disease heterogeneity with nanomedicine, which is the major reason for the failures observed in translation of promising nanoformulations in clinical trials. Rigorous evaluation of nanoformulations in multiple preclinical animal models is necessary before the onset of clinical trials. Preclinical studies in animal models often provide useful information toward the suitability of theranostic nanomedicine for treating and imaging human patient groups [22]. During the early stages of clinical development, nanotoxicology profiles comprising of standardized assay protocols for cytotoxicity, immunotoxicity and genotoxicity need to be implemented and followed for the evaluation of the potential risk in patients [15,23].

Development of nanotheranostics for diseases other than cancer must be emphasized by exploring major properties besides enhanced permeability and retention effects, as noncancerous diseases mostly do not possess impaired vascular anatomy. Therefore, development of biomimetic nanoparticles for exploiting the natural functionality of the source cell (like secretory molecules, cell surface marker, extra cellular matrix, etc.) will provide huge potentiality for theranostic applications. The real-life impact of theranostics can further be augmented by using stimuli-based smart nanoparticles for the release of the therapeutic load exclusively at the site of action when necessary. This kind of provision and real-time monitoring can help the clinician to adjust the treatment plan in a personalized manner for diseases showing heterogeneity, malignancy and adaptation.

Nanoparticle-based drug-delivery systems are originating from academic laboratories, which usually function on a small scale, emphasizing on new scientific and technological developments. They are often familiar with the technical challenges faced by industry in commercializing processes. To bridge this gap, strong collaboration between laboratory groups and pharmaceutical companies needs to be established. Modified rules under good manufacturing practice that are suitable for large-scale synthesis of theranostic nanoparticles must be developed. Further, the use of process optimization software such as Aspen (AspenTech, MA, USA) in industrial setting can be of great help to identify the key parameters to optimize manufacturing at the early stages of development and handle batch-to-batch variation. This may be beneficial for tightly controlled and robust manufacturing of the product [24]. At last, the success of manufacturing is also highly dependent on the training of personnel regarding the specificities, hurdles and challenges related to the products. Undoubtedly, theranostic nanomedicines can influence human health in the clinic today but applying the above lessons in the early stages of development can prepare the producers to develop efficient products.

\section{Conclusion}

Theranostic nanomedicine has evolved considerably and shows remarkable advancement, still, the field has to mature before it can revolutionize the field of human medicine. Nanotheranostics is an add-on concept in nanomedicine and could find numerous application-oriented opportunities in clinics for disease management. Therefore, it is required to break the dogma and explore the possibilities to make theranostic nanomedicine a great success for human use. It is clear that despite the promise of nanotheranostics, improvement in our understanding of interwoven therapeutic and diagnostic agents is required to enhance its potential for clinical application. Further, the most promising approaches need to be followed at both commercialization and regulatory steps for bringing theranostic nanomedicine from research laboratories to clinics for patient's use.

\section{Financial \& competing interest disclosure}

The authors have no relevant affiliations or financial involvement with any organization or entity with a financial interest in or financial conflict with the subject matter or materials discussed in the manuscript. This includes employment, consultancies, honoraria, stock ownership or options, expert testimony, grants or patents received or pending, or royalties.

No writing assistance was utilized in the production of this manuscript. 


\section{References}

1. Chen H, Zhang W, Zhu G, Xie J, Chen X. Rethinking cancer nanotheranostics. Nat. Rev. Mater. 2, 17024 (2017).

2. Silva CO, Pinho JO, Lopes JM, Almeida AJ, Gaspar MM, Reis C. Current trends in cancer nanotheranostics: metallic, polymeric, and lipid-based systems. Pharmaceutics 11(1), 1-40 (2019).

3. Pedrosa P, Vinhas R, Fernandes A, Baptista PV. Gold nanotheranostics: proof-of-concept or clinical tool? Nanomaterials (Basel) 5(4), 1853-1879 (2015).

4. Roy Chowdhury M, Schumann C, Bhakta-Guha D, Guha G. Cancer nanotheranostics: strategies, promises and impediments. Biomed. Pharmacother. 84, 291-304 (2016).

5. Lammers T, Aime S, Hennink WE, Storm G, Kiessling F. Theranostic nanomedicine. Acc. Chem. Res. 44(10), 1029-1038 (2011).

6. Kim M, Jang J, Cha C. Carbon nanomaterials as versatile platforms for theranostic applications. Drug Discov. Today 22(9), 1430-1437 (2017).

7. Ding K, Jing L, Liu C, Hou Y, Gao M. Magnetically engineered Cd-free quantum dots as dual-modality probes for fluorescence/magnetic resonance imaging of tumors. Biomaterials 35(5), 1608-1617 (2014).

8. Park SM, Aalipour A, Vermesh O, Yu JH, Gambhir SS. Towards clinically translatable in vivo nanodiagnostics. Nat. Rev. Mater. 2(5), 17014 (2017).

9. Mura S, Couvreur P. Nanotheranostics for personalized medicine. Adv. Drug Deliv. Rev. 64(13), 1394-1416 (2012).

10. Yong $\mathrm{Y}$, Zhou L, Zhang S et al. Gadolinium polytungstate nanoclusters: a new theranostic with ultrasmall size and versatile properties for dual-modal MR/CT imaging and photothermal therapy/radiotherapy of cancer. Npg Asia Mater. 8, e273 (2016).

11. Jung E, Kang C, Lee J et al. Molecularly engineered theranostic nanoparticles for thrombosed vessels: $\mathrm{H}_{2} \mathrm{O}_{2}$-activatable contrast-enhanced photoacoustic imaging and antithrombotic therapy. ACS Nano 12(1), 392-401 (2018).

12. Dilnawaz F, Acharya S, Sahoo SK. Recent trends of nanomedicinal approaches in clinics. Int. J. Pharm. 538(1-2), $263-278$ (2018).

13. Riehemann K, Schneider SW, Luger TA, Godin B, Ferrari M, Fuchs H. Nanomedicine - challenge and perspectives. Angew. Chem. Int. Ed. Engl. 48(5), 872-897 (2009).

14. Shi J, Kantoff PW, Wooster R, Farokhzad OC. Cancer nanomedicine: progress, challenges and opportunities. Nat. Rev. Cancer 17(1), 20-37 (2017).

15. Jackman JA, Meszaros T, Fulop T, Urbanics R, Szebeni J, Cho NJ. Comparison of complement activation-related pseudoallergy in miniature and domestic pigs: foundation of a validatable immune toxicity model. Nanomedicine 12(4), 933-943 (2016).

16. Szebeni J, Storm G. Complement activation as a bioequivalence issue relevant to the development of generic liposomes and other nanoparticulate drugs. Biochem. Biophys. Res. Commun. 468(3), 490-497 (2015).

17. Hua S, de Matos MBC, Metselaar JM, Storm G. Current trends and challenges in the clinical translation of nanoparticulate nanomedicines: pathways for translational development and commercialization. Front. Pharmacol. 9, 790 (2018).

18. Dreifuss T, Betzer O, Shilo M, Popovtzer A, Motiei M, Popovtzer R. A challenge for theranostics: is the optimal particle for therapy also optimal for diagnostics? Nanoscale 7(37), 15175-15184 (2015).

19. Sainz V, Conniot J, Matos AI et al. Regulatory aspects on nanomedicines. Biochem. Biophys. Res. Commun. 468(3), 504-510 (2015).

20. Gaspar R. Regulatory issues surrounding nanomedicines: setting the scene for the next generation of nanopharmaceuticals. Nanomedicine (Lond.) 2(2), 143-147 (2007).

21. Tinkle S, McNeil SE, Muhlebach S et al. Nanomedicines: addressing the scientific and regulatory gap. Ann. NY Acad. Sci. 1313, 35-56 (2014).

22. Hare JI, Lammers T, Ashford MB, Puri S, Storm G, Barry ST. Challenges and strategies in anti-cancer nanomedicine development: an industry perspective. Adv. Drug Deliv. Rev. 108, 25-38 (2017).

23. Accomasso L, Cristallini C, Giachino C. Risk assessment and risk minimization in nanomedicine: a need for predictive, alternative, and 3Rs strategies. Front. Pharmacol. 9, 228 (2018).

24. Ragelle H, Danhier F, Preat V, Langer R, Anderson DG. Nanoparticle-based drug delivery systems: a commercial and regulatory outlook as the field matures. Expert Opin. Drug Deliv. 14(7), 851-864 (2017). 